\title{
LAS ESFERAS MULTIPARTIDA COMO SISTEMA DE REGISTRO CONTABLE GERENCIAL, GENERACIÓN 2021
}

\author{
THE SPHERES MULTIBOOKKEEPING AS A MANAGEMENT ACCOUNTING \\ RECORD SYSTEM, GENERATION 2021
}

Carlos LoZano NúÑEZ Universidad Nacional de Huancavelica Huancavelica, Perú ORCID: https://orcid.org/0000-0001-6871-7110 Correo electrónico: carlos.lozano@unh.edu.pe

Pedro Demetrio Lozano Núñez Universidad Nacional del Centro del Perú Huancayo, Perú ORCID: https://orcid.org/0000-0003-2049-1807 Correo electrónico: plozanon@hotmail.com

Ada Lina Mercado Guillén I.E. N 31942 Mariscal Castilla Huancayo, Perú

ORCID: https://orcid.org/0000-0002-2004-0021 Correo electrónico: adamercadog@hotmail.com

[Recibido: 21/01/2021 Aceptado: 27/04/2021 Publicado: 27/05/2021]

\section{RESUMEN}

Objetivo: Analizar la evolución de las partidas contables y proponer un reporte de registro contable gerencial denominado esfera multipartida de generación 2021. Método: Investigación no experimental y descriptiva. Se analizaron artículos de investigación, publicaciones de memorias de congresos y eventos contables. Se consideraron las opiniones de 100 personas que efectuaron compras de productos en una empresa. Resultados: La esfera de partida doble es un sistema de registro frío, mecánico, tradicional y rutinario; se mantiene vigente desde hace más de 500 años, presenta limitaciones de medición contable, de registro y decisional. El reporte propuesto de esferas multipartida presenta componentes de esferas de partida triple, partida cuádruple, pentapartida, al infinito como sistemas de registros contables compuesto por " $n$ " dimensiones de variables cuantitativas y cualitativas internas o externas que afectan a la empresa. Estas son valorizadas y puntuadas de forma positiva o negativa, expresadas en diversos indicadores para medir los eventos, sucesos y los hechos contables del entorno empresarial. Se construyen y se adecuan por constructos que gestiona el Contador Público. Conclusión: El reporte de esferas multipartida como registro contable gerencial de generación 2021 permite medir variables cualitativas y cuantitativas internas o externas que afectan a la empresa, y requiere altas competencias del Contador Público.

Palabras clave: Esferas multipartida; registros contables; reporte gerencial; dimensión contable; gestor contable.

\begin{abstract}
Objective: To analyze the evolution of accounting bookkeeping and propose a management accounting record report denominated multi-bookkeeping sphere of generation 2021. Method: The method used was the descriptive and non-experimental research. In which a series of research articles, congress memory publications and countable events were analyzed. In addition, a sample of 100 people's opinions, regarding their experience shopping in a company, was also considered for the research. Results: The double bookkeeping sphere is a cold, mechanic, traditional and routine register system; it has been kept in force for more than 500 years, it presents limitations as are the managerial countable measurement, registration and decision making process. The proposed multi-bookkeeping spheres' report presents components of triple, quadruple, quintuple to infinity bookkeeping's spheres as systems of accounting records composed by " $n$ " dimensions of qualitative and quantitative variables, which are also internal or external and affect the company. These are rated and counted either in a positive or negative form, and expressed in various indicators to measure the events and the countable facts of the business' environment. Also, they are built and adapted by constructs managed by the public accountant. Conclusion: The report of multi-bookkeeping spheres as the managerial accounting record of generation 2021, allows measuring the internal or external quantitative and qualitative variables that affect the company, and requires the public accountant's competences.
\end{abstract}

Keywords: Spheres multibookkeeping; accounting records; managerial report; accounting dimension; countable manager. 


\section{INTRODUCCIÓN}

La evolución de las esferas partida simple, partida doble, partida triple, hacia las "n" esferas denominadas esfera multipartida, representa la realidad compleja de los sistemas de registros utilizados por la contabilidad a través de la historia. Estos últimos permiten la captación y valoración de la realidad económica mediante el análisis y la interpretación inductiva de la información revelada en términos contables, siendo inexpresiva y desordenada con una simple observación (Ortiz, 2013). Puesto que, al tener limitaciones de registro y medición de variables cuantitativas y cualitativas, requiere el cambio de paradigmas hacia las esferas multipartida integral para satisfacer las necesidades evolutivas de información, de registro y medición contable.

Se fundamenta en que Ijiri (1986) demostró que "la contabilidad de doble entrada no es un sistema absoluto que desafíe las extensiones, pero es lógicamente extensible a la contabilidad de triple entrada" (p. 745), dado que "la partida doble muestra sus limitaciones a la hora de describir el capitalismo actual. Se corre el gran riesgo de tomar importantes decisiones económicas basadas en una forma de representación pobremente adaptada a lo que se quiere describir" (Corvellec, 2001, p. 8).

Kaplan (1984) fundamenta que el investigador que nunca se lanza al agua, que recolecta datos cuantitativos a distancia, sin anécdotas que soporten ellos, siempre tendrá dificultades para explicar relaciones interesantes de nuestros procesos mentales subconscientes y nuestra intuición. Siendo necesario "seleccionar adecuadamente las variables relevantes que caracterizan los fenómenos tratados, definir la unidad de medida adecuada, elaborar y/o perfeccionar los instrumentos de medición que permitan captar las propiedades del fenómeno analizado" (Fernández y Barbei, 2006, p. 83). Sin embargo, Fraser (1993) refiere que "la adición de una tercera dimensión no mejorará esta situación” (p.153).

“De modo semejante, más y más empresas en los últimos años han escogido completar su contabilidad tradicional con una descripción multidimensional de su actividad" (Corvellec, 2001, p. 7). Hechos descritos que fundamentan y evidencian las necesidades evolutivas del explicans y el explicandum teórico y práctico de los sistemas de registros contables hacia el cambio de paradigmas del Contador Público a partir de la generación 2021.

Las esferas de registro y medición contable se inician con la esfera partida simple, debido al intenso movimiento mercantil de las repúblicas que existían en la Italia septentrional. Estos hechos permitían llevar la cuenta y razón de modo simple de las operaciones mercantiles mediante un simple registro de la actividad de los negocios anotados por los comerciantes de aquel entonces por diferentes conceptos sobre las mercaderías, y no requerían la experticia de un contador (Boter, 1959). La debilidad de este sistema de registro unidireccional no permitía contraponer mediante una contrapartida para un mejor registro y control.

La esfera partida doble evoluciona de los sistemas de registros contables, fue utilizada por los mercaderes italianos que registraban en sus libros de un modo primigenio las operaciones o hechos contables mediante un sistema de contrapartida denominado modo de Venecia, el cual consistía en el registro bidireccional del cargo y abono y/o el débito y crédito (Boter, 1959). Los primeros hallazgos de las muestras de partida doble fueron en la contabilidad que llevaban los mercaderes de los estados italianos en la edad media (Villaluenga, 2013).

La esfera de "partida doble" se mantiene vigente hasta la actualidad habiendo trascurrido ya más de 500 años. "La amplitud y relevancia de este proceso, requiere que se fundamente adecuadamente, a fin de poder realizar mediciones de fenómenos contables sobre bases rigurosas, compartiendo un marco teórico conceptual" (Fernández y Barbei, 2006, p. 83).

La contabilidad tradicional por partida doble, al registrar y medir un asiento contable, es fría, mecánica y cuantitativa. Por ejemplo, no refleja la capacidad de gestión del gerente, la eficiencia del vendedor, la atención recibida entre otros de carácter cualitativo. "Siendo imperfectos y débiles para tomar decisiones. Los elementos tradicionales (gnoseológicos, conceptuales, técnico-procedimentales) deben ser redefinidos para ampliar el espectro teórico aplicado de la evaluación de la gestión de las organizaciones" (Mejía et al., 2013, p. 50). "La medición contable no es exclusivamente de valores económicos, sino de valores humanos individuales y sociales" (Mejía, Montes, y Dávila, 2011, p. 127).

La medición contable, en un sentido amplio, abarca la dimensión cualitativa y la cuantitativa y no se limita a las mediciones monetarias, pues utiliza diversas escalas y unidades de medida (Mejía et al., 2011, p. 159). "El estudio sistemático de la Contabilidad se puede hacer con muchos instrumentos" (Mejía E. et al., 2011, p. 128), y requiere "seleccionar adecuadamente las variables relevantes que caracterizan los fenómenos tratados, definir la unidad de medida adecuada, elaborar y/o perfeccionar los instrumentos de medición que permitan captar las propiedades del fenómeno analizado" (Fernández y Barbei, 2006, p. 83). 
"La actitud competitiva será mejor si nosotros los productores de esa nueva realidad logramos anticiparnos y reaccionar a los cambios del ambiente, utilizando la capacidad e ingenio creativo para generar nuevas ideas" (Velásquez, 2019, pp. 34-35). Basado en ello, la labor del Contador Público se hace necesaria y primordial para gestionar las esferas de los registros y las mediciones contables.

En este contexto, no solo adquiere relevancia la necesidad evolutiva de los registros contables, sino su interacción con el ambiente interno y externo empresarial donde el desempeño funcional del gestor contable en la organización es esencial y fundamental. "El modo en que se usa la información generada por el sistema contable impacta de modo diferente en el desempeño organizacional, especialmente en ámbitos turbulentos como en Latinoamérica" (Porporato, 2015, p. 512).

En la esfera de partida triple, Ijiri (1986) refiere que es la extensión de partida doble hacia la partida triple en razón que, la contabilidad sólo registraba hechos contables de activos y pasivos. Luego se amplió a partida doble considerando las cuentas de flujos como las cuentas de ingresos y gastos, y que para pasar a partida triple debe contener tres categorías de dimensiones contables: a) riqueza en la primera columna (débito), b) Impulso e ingresos en la segunda columna (crédito) y c) fuerza, impulso y acción en la tercera columna (trébito).

Se ha evidenciado que en la investigación realizada por Ijiri (1986) se presentan razonamientos lógicos y teóricos con ejemplos simples. En la esfera partida triple las tres columnas tienen la misma medida y están articuladas, existen factores en la tercera columna que explican los cambios en la primera y segunda columnas. No obstante, se menciona que, "La limitación de este estudio es que los pensamientos de Ijiri no se han implantado y, por lo tanto, no se ha realizado ninguna prueba empírica para examinar la eficacia de estos inventos" (Pei-Gin, 2013, p. 51). En las esferas multipartida se refuta este razonamiento, pues cada columna representa una dimensión de contrapartida que representa el registro y medición de variables e indicadores cuantitativos y cualitativos.

Las gradas evolutivas de las esferas de registro contable continúan mediante un modelo de partida triple que consiste en un sistema de registro tridimensional. El cual permite medir y evaluar los indicadores de cada hecho contable de una organización empresarial o de los negocios a nivel gerencial mediante ponderaciones para cada una de las dimensiones, estableciendo ejemplos significativos para las tres columnas (débito, crédito y trébito). (Lozano, C. y Lozano P., 2012). De esta manera, es necesario conocer y controlar los movimientos de ingresos y egresos provenientes de las actividades de operación, inversión y financiamiento de la empresa mediante el flujo de caja (Moreno et al.,2017).

"Todas las reflexiones anteriores desembocan en la creación de la contabilidad triangular o de partida triple" (Arjona, 2012, p. 27). El planteamiento de partida triple expresado por Arjona se refería a una tercera columna que debería tratar sobre el flujo del efectivo, siendo diferente a la propuesta que incluye dimensiones de variables o razones, y se mide mediante indicadores, pero invoca ya un cambio de paradigmas.

Entonces si existe una primera, segunda y tercera columna, entonces existen " $n$ " columnas para un sistema de registro contable que soporte y fundamente la extensión de partidas contables hacia la esfera multipartida. "Se comprenderá fácilmente porque desde la década de los ochenta del siglo XX sobre todo en los países industrializados se empezó a vislumbrar la necesidad de adecuar las profesiones existentes a las nuevas exigencias del entorno" (Damián, 2018, p. 458).

La esfera partida cuádruple propuesta por Postner (1998) complementa la evolución de las esferas de registros contables. En ella se muestran ejemplos que típicamente involucran transacciones económicas "complejas" entre unidades de negocios, en las que la forma legal de las transacciones no necesariamente representa su sustancia económica. La representación de partida cuádruple "vincula la micro y macro contabilidad para la cuenta nacional" (Staszkiewicz, 2011, p. 6).

Actualmente, la esfera multipartida representa la necesidad evolutiva de los sistemas de registros contables a nivel gerencial hacia un sistema de registro y medición integral. La conjetura doctrinaria parte de la semejanza de la empresa con un árbol contable que simboliza las partidas contables en el registro de las operaciones y hechos contables de la empresa: una rama permite el registro por partida simple, dos ramas, permite el registro por partida doble, tres ramas, permite el registro por partida triple y varias ramas permiten el registro por partida multidimensional (Lozano, C. y Lozano, P., 2012).

El término multidimensional se refiere a varias dimensiones o esferas utilizadas para el registro de los hechos contables. En la física "Una dimensión es una propiedad del espacio físico definida por el número de coordenadas necesarias para especificar la medida de un objeto" Sheposh (2019). En las ciencias exactas "Si consideramos como noción de tamaño a la dimensión de Hausdor, hay conjuntos grandes sin ciertos patrones, como así también conjuntos chicos con muchos patrones. Otra noción de tamaño importante es el espesor, definido por Newhouse" (Yavicoli, 2019 , p. 2). En la contabilidad una dimensión es aquella 
que tiene "una estructura aplicativa que permite medir y evaluar simultáneamente las ' $\mathrm{n}$ ' dimensiones (reflectos) de operaciones meta contables mediante ponderaciones que se presentan en cada transacción, estas son explicadas mediante 'razones' sustentadas con documentación y análisis fuente a nivel gerencial" (Lozano, 2019, p. 91). "La materialización y concreción cualitativa y cuantitativa de esas dimensiones, mediante la explicitación de una serie de aspectos y facetas de las mismas se ha ido proyectando en una serie de indicadores representativos al respecto" (Lizcano, 1982, p. 437).

"La metodología de aplicación y de gestión de la partida multidimensional requiere la medición mediante la escala de valoración que se aplican en el proceso decisional racional" (Lozano, 2019, p. 215). Esta permite modelar fenómenos estabilizadores como alternativa de registro. También, para el análisis, constructo e interpretación de la partida multidimensional, se requiere un gestor de la información y el conocimiento contable denominado Contador Público.

La esfera multipartida requiere modelar fenómenos estabilizadores, se necesitan impulsos de formatos diferentes a los índices como rendimiento de los activos o del capital que proporcionan señales informativas importantes al analista de los estados financieros (Melse, 2008). "Su constructo requiere partir de las variables: la bibliografía, opinión de directivos empresariales" (Bernal, 2011). La elaboración aplicabilidad de un modelo propio requiere del conocimiento, habilidades y destrezas del Contador Público y la utilización base de la tabla 1. Damián (2018) refiere que, "algunas profesiones tradicionales necesitan reinventarse para continuar generando oportunidades de trabajo" (p. 458)

La contabilidad, que mantiene sistemas de registros tradicionales, necesita reinventarse con la propuesta de las esferas multipartida que considere "La dimensión contable administrativa, social y económica que permita identificar y sustentar usuarios de la información y necesidades de información superando la visión del usuario inversor proveedor de capital de riesgo" (Mejía et al. ,2011, p. 133). Túa al citar a Mattessich refiere que en la práctica real pueden encontrarse sistemas diferentes a lo prescrito en la teoría general que al contrastarse en la realidad puede refutarse o excluirse (Mejía et al., 2005, pp. 102-103).

Galle y Mucelli (2015) refieren que, para afrontar el entorno dinámico empresarial futuro, se exigirá asociar las variables endógenas con las exógenas dado que el entorno es incierto, cambiante y complejo en aspectos financieros, económicos y patrimoniales donde es necesario utilizar lupas para detectar y comprender procesos minúsculos en la empresa.
Finalmente, el sistema de registro contable mediante esferas de partida triple y multipartida, permite registrar los hechos y fenómenos contables en la gestión empresarial. Asimismo, se requiere lograr el cambio de paradigmas del Contador Público y dar los saltos evolutivos paradigmáticos de esferas de registro contable hacia la esfera multipartida integral.

\section{MATERIALES Y MÉTODOS}

La investigación fue no experimental, documental y descriptiva. Se analizaron artículos indexados de investigación y publicaciones de memorias de congresos y eventos contables, registrando el marco conceptual evolutivo del sistema de registros contables según autores referenciados. Se consideraron opiniones de 100 personas que efectuaron compras de productos en una empresa, el instrumento utilizado fue un registro de opiniones. Mediante el método científico, se han analizado críticamente las restricciones de los sistemas de registro tradicional por partida doble y simple vigentes desde hace más de 500 años, cómo han ido evolucionando a través del tiempo mediante esferas de multipartida, y cómo estás afectan el entorno interno y externo de la empresa mediante el resultado esperado. Se analizó, mediante la técnica de opiniones de la muestra intencionada, a 100 personas que compraron productos de la empresa, luego se ha abstraído y construido el reporte multipartida de las esferas evolutivas de los sistemas de registro como Contador Público en calidad del gestor de la información y el conocimiento contable. Las fases investigativas fueron: 1. Recopilación y análisis de los materiales utilizados: los comprobantes de pago y las propuestas del reporte multipartida, 2. Determinación de las esferas de registro, precisión de las multidimensiones (evidencia de registro) y 3. Determinación de los indicadores, valorización positiva y negativa, puntuación positiva y negativa.

\section{RESULTADOS}

En la Figura 1, esferas multipartida de la empresa, se muestra los sistemas de registro contable iniciando desde la "partida simple", "partida doble" denominados tradicionales y la "partida triple", "partida cuádruple", "pentapartida", "hexapartida", "heptapartida" y la "octopartida" denominados esferas evolutivas que representan las dimensiones e indicadores originados por el registro contable de las ventas del producto " $\mathrm{X}$ " en la empresa, $\mathrm{y}$ determinado por el gestor contable denominado Contador Público.

Aplicación práctica: La gerencia desea saber que dimensiones, indicadores, montos y puntajes negativos o positivos se genera al realizar una venta de S/ 1000 del producto "X" de la empresa. Utilizar el reporte multipartida. 


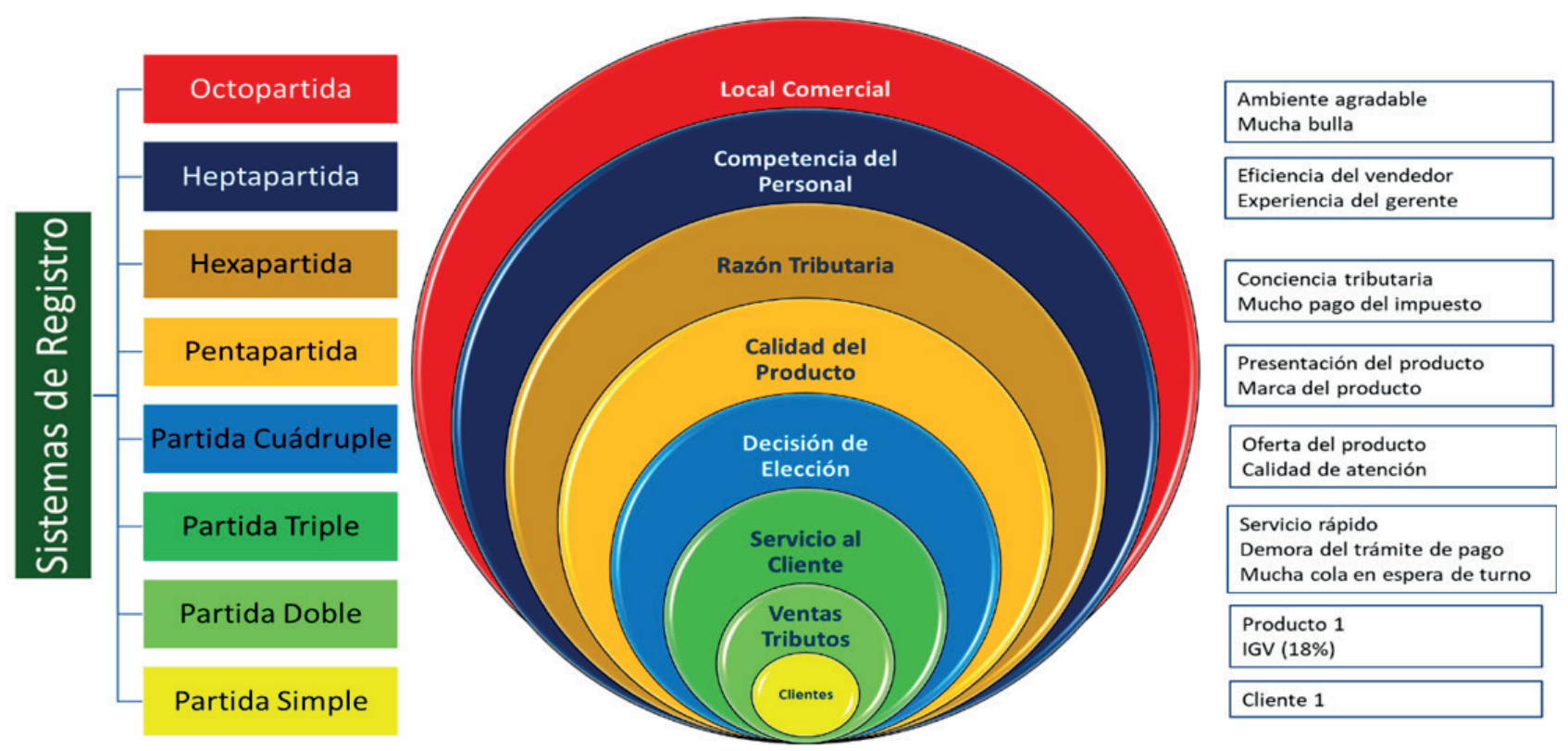

Figura 1. Esferas multipartida de la empresa

Fuente: Elaboración propia a partir del constructo multipartida

El reporte multipartida de la empresa 2020 (ver tabla 1) está compuesto por tres columnas: Registro contable multipartida (esferas, dimensión e indicadores), valorización (positiva y negativa) y calificación (positiva y negativa). Esferas que representan el registro contable de las partidas utilizadas: partida doble, partida triple, partida cuádruple, pentapartida, heptapartida y la octopartida.

Las dimensiones/indicadores utilizados para medir cada esfera de partidas contables son: El asiento contable de ventas tradicional (clientes/ventas-tributo), el servicio al cliente (demora en el trámite y mucha cola), la decisión de elección (calidad de atención, oferta y servicio), la calidad del producto (marca y presentación), la razón tributaria (pago elevado y conciencia tributaria), competencia del personal (experiencia del gerente y eficiencia del vendedor) y el local comercial (mucha bulla y ambiente agradable).

La valorización está representada por las cantidades de dinero a partir de la esfera partida doble, dinero que se origina por ingresos (ganancias + ) y egresos (pérdidas -) a la empresa. Esta la valoriza el gestor contable a partir de la información recibida, su experiencia y su experticia valorativa.

La calificación representa la ponderación del puntaje positivo o negativo que asigna el cliente. Ejemplo ventas del producto " $\mathrm{x}$ " por S/ 1,000 para la esfera partida triple se compone de tres columnas que registra:
1) Débito: se registra el asiento tradicional: clientes $S / 1$ 180 (primera columna-esfera partida simple), 2) Abono: se registra el asiento tradicional: Ventas S/ 1000 y tributos S/ 180 (segunda columna- esfera partida doble) y 3) Trébito: se registra por partida triple, el servicio al cliente tercera dimensión (tercera columna - esfera partida triple) que el gestor contable realiza la valorización como trébito negativo por demora en el pago por S/ 870 $(870 / 2,781=31.28 \%)$ y mucha cola de espera por S/ 1326 $(1,326 / 2,781=47 \cdot 68 \%)$.

El puntaje de la calificación negativa que realizan los clientes asciende a $145(145 / 420=34.52 \%)$, por ejemplo, son 29 clientes que cada uno calificó -5 puntos negativos por la demora en el trámite y mucho tiempo de espera. Por lo tanto, mediante el reporte multipartida se observa que la empresa tuvo ingresos reales por S/ 4384 y egresos reales por $S / 2$ 781, generando una medición de los puntajes positivos de 1,519 y negativos de 420 .

Del análisis y la medición de la Figura 1: Esferas multipartida de la empresa, y la tabla 1: Reporte multipartida al 31 de diciembre del 2020 de la empresa se concluye que, el simple registro de la partida doble tradicional del cargo: clientes S/ 1180 y el abono ventas S/ 1000 y tributo S/ 180 no mide lo que realmente debe medir y no considera las dimensiones y los indicadores que afectan el hecho contable del registro de una venta en la empresa, siendo 
decisivo e imprescindible el conocimiento y experiencia del gestor contable denominado Contador Público.

\section{DISCUSIÓN}

Las empresas del país requieren un sistema de registro contable que se adapte al entorno cambiante; es así que la propuesta de la esfera multipartida como herramienta de registro y control gerencial es una herramienta que surge como alternativa para los Contadores Públicos frente a las limitaciones de la contabilidad tradicional de registro por partida doble.

En la actualidad se sigue utilizando, pero no satisface por ser fría, mecánica y tradicional. A través del paso del tiempo, cada vez existen más restricciones para brindar información cuantitativa y cualitativa en un entorno de cambios constantes en el ambiente empresarial influenciados por las variables tecnológicas, económicas, sociales, culturales, políticas y educativas. Se sustenta porque el sistema de registro contable tradicional no mide ni valoriza las variables del entorno que afectan a la empresa, basándose solo en el plan contable empresarial, el cual es imperfecto y débil para la toma de decisiones. Es por ello que se concuerda con Porporato (2015), que refiere el modo en que se usa la información generada por el sistema contable y como impacta es de modo diferente en la empresa. En el caso por partida doble, el impacto es nulo.

En la esfera partida triple Glover (2018) refiere que Ijiri es teórico, la partida triple es difícil de clasificar, y aconseja diseñar un modelo que incluya nuevas cuentas y nuevas reglas contables; basándose en muchos factores, puesto que presenta limitaciones de medición dimensional. Por lo tanto, es diferente al modelo propuesto de esfera multipartida, diferenciándose desde su óptica teórica y por su aplicabilidad práctica (ver tabla 1).

Tabla 1

Reporte multipartida al 31 de diciembre del 2020 de la empresa

\begin{tabular}{|c|c|c|c|c|c|c|c|c|c|c|}
\hline \multicolumn{3}{|c|}{ Registro contable multipartida } & \multicolumn{2}{|c|}{ Valorización + } & \multicolumn{2}{|c|}{ Valorización - } & \multicolumn{2}{|c|}{ Calificación + } & \multicolumn{2}{|c|}{ Calificación - } \\
\hline Esferas & Dimensión & Indicadores & $\mathrm{S} /$ & $\%$ & $\mathrm{~S} /$ & $\%$ & Puntaje & $\%$ & Puntaje & $\%$ \\
\hline \multirow[t]{2}{*}{ Octopartida } & \multirow[t]{2}{*}{ Local comercial } & Mucha bulla & & & 330 & 11.87 & & & 90 & 21.43 \\
\hline & & Ambiente agradable & 84 & 1.92 & & & 98 & 6.45 & & \\
\hline \multirow[t]{2}{*}{ Heptapartida } & \multirow{2}{*}{$\begin{array}{l}\text { Competencia del } \\
\text { personal }\end{array}$} & Experiencia del gerente & 300 & 6.84 & & & 55 & 3.62 & & \\
\hline & & Eficiencia del vendedor & 2,100 & 47.9 & & & 540 & 35.55 & & \\
\hline \multirow[t]{2}{*}{ Hexapartida } & \multirow[t]{2}{*}{ Razón tributaria } & Mucho pago del impuesto & & & 255 & 9.17 & & & 68 & 16.19 \\
\hline & & Conciencia tributaria & 30 & 0.68 & & & 78 & 5.13 & & \\
\hline \multirow[t]{2}{*}{ Pentapartida } & \multirow{2}{*}{$\begin{array}{l}\text { Calidad del } \\
\text { producto }\end{array}$} & Marca del producto & 210 & 4.79 & & & 72 & 4.74 & & \\
\hline & & Presentación del producto & 80 & 1.82 & & & 96 & 6.32 & & \\
\hline \multirow[t]{2}{*}{ Partida Cuádruple } & \multirow{2}{*}{$\begin{array}{l}\text { Decisión de } \\
\text { elección }\end{array}$} & Calidad de atención & 100 & 2.28 & & & 60 & 3.95 & & \\
\hline & & Oferta del producto & 880 & 20.07 & & & 352 & 23.17 & & \\
\hline \multirow[t]{4}{*}{ Partida Triple } & Servicio al cliente & Servicio rápido & 600 & 13.69 & & & 168 & 11.06 & & \\
\hline & & Demora trámite en el pago & & & 870 & 31.28 & & & 145 & 34.52 \\
\hline & & Mucha cola en espera de turno & & & 1,326 & 47.68 & & & 117 & 27.86 \\
\hline & & Sub -Total: Evolutivo & 4,384 & 100 & 2,781 & 100 & 1,519 & 100 & 420 & 100 \\
\hline \multirow[t]{5}{*}{ Partida Doble } & Clientes & Cliente 1 & 1,180 & & & & & & & \\
\hline & Ventas & Producto 1 & & & 1,000 & & & & & \\
\hline & Tributo & IGV (18\%) & & & 180 & & & & & \\
\hline & & Sub -Total: Tradicional & 1,180 & & 1,180 & & & & & \\
\hline & & Total & 5,564 & 100 & 3,961 & 100 & 1519 & 100 & 420 & 100 \\
\hline
\end{tabular}

Nota: La tabla 1 representa un constructo de reporte multipartida para la empresa compuesto de esferas de partidas de registro contable con sus variables o dimensiones con indicadores cualitativos o cuantitativos sean internos o externos valorizados y puntuados (+) y (-). La esfera de partida simple y la partida doble representan el registro contable tradicional y a partir de la esfera partida triple representa las partidas evolutivas contables.

Fuente: Elaboración propia a partir del constructo de las variables e indicadores del entorno empresarial 
Se ha evidenciado que los ejemplos de Ijiri (1986) son teóricos, cada dimensión incorpora la riqueza, el impulso y la fuerza, no son separados por columnas y no ha realizado ninguna prueba empírica de estos constructos (Pei-Gin Hsieh, 2013). Se concuerda con Moreno et al. (2017) que refiere que ha sido necesaria la evolución de la contabilidad mediante la propuesta de la partida triple.

El reporte de esferas multipartida comparada con la partida triple, mencionado por Arjona (2012) al referirse a una tercera columna sobre el flujo del efectivo, no abarca las variables del entorno. Razones suficientes expuestas por Damián (2018) que refiere que, algunas profesiones tradicionales, como es el caso de la contabilidad, necesitan reinventarse para continuar generando oportunidades de trabajo.

El Contador Público de hoy, en su calidad como gestor contable, requiere una herramienta que sea útil, adaptable y que sirva para realizar escenarios donde la empresa identifique que variables internas o externas están afectando a la empresa.

Mediante un simple registro por partida doble de la transacción comercial no se puede medir ni registrar el hecho contable que involucra variables como el esfuerzo del gerente, la eficiencia del vendedor, qué indicadores son buenos o positivos, qué aspectos del entorno afectan las ventas de la empresa o cuáles son los factores negativos donde se puede identificarlos, valorarlos, determinar su puntuación y que en conjunto permita una toma de decisiones.

La esfera partida cuádruple referida por Postner (1998), en sí, no es tan compleja o difícil, dado que el sistema de registro y medición por esferas multipartida involucra transacciones económicas en cuatro columnas (dimensiones). Staszkiewicz (2011) explica que ya existe una entrada cuádruple, la cual se considera necesaria y se puede visualizar en la figura 1 y la tabla 1 , instrumento presentado en calidad de reporte que facilita y permite medir y registrar, no solo la cuarta columna como dimensión contable, sino que incluye también los indicadores, sus valorizaciones y puntuaciones aplicados a una realidad empresarial.

$\mathrm{El}$ análisis mediante el constructo del reporte multipartida (ver tabla 1), representado por cada esfera de partidas como registros contables, conlleva a formular las decisiones y acciones estratégicas para la empresa debiendo potenciar aún más la eficiencia de los vendedores de la empresa para generar más valorizaciones positivas evitando las negativas.

Actualmente, la nueva generación 2021 de Contadores Públicos requiere conocer y dar saltos de paradigmas de las esferas de registros contables para hacer frente al entorno cambiante, razón por la cual se propone el uso y gestión del reporte de esferas multipartida por su aplicabilidad y demostración práctica (tabla 1).

Se concluye que, el reporte de esferas multipartida como registro contable gerencial de generación 2021 permite medir variables cualitativas y cuantitativas internas o externas que afectan a la empresa, y requiere altas y significativas competencias del Contador Público en su calidad de gestor del conocimiento y de la información contable. Las limitaciones del estudio son, las escasas investigaciones de fuentes teóricas y aplicaciones prácticas, estandarizar los reportes gerenciales para la toma de decisiones y la simulación de escenarios mediante la esfera evolutiva multipartida.

\section{REFERENCIAS}

Arjona, A. (2012). La contabilidad triangular (o de partida triple). Alicante: Editorial Club Universitario.

Bernal, C. A. (2011). Modelo de gerencia del conocimiento: visión integral. Cuadernos de Administración, 27(46), 55-66. Recuperado de: http://www.redalyc. org/articulo.oa?id=225022711005

Boter, F. (1959). Las doctrinas contables. Barcelona: Editorial Juventud.

Corvellec, H. (2001). La contabilidad de partida doble como forma de representación. Heterogénesis. Revista de Artes Visuales, 36. Recuperado de: http://www.redalyc.org/pdf/108/10803602.pdf

Damián, J. (2018). Yo estudio para ser ingeniero: ¿para qué cursar administración y contabilidad? Propósitos y Representaciones, 6(2), 453-497. doi: https://dx.doi. org/10.20511/pyr2018.v6n2.227

Fernández, L. y Barbei, A. (2006). La medición en contabilidad: Un análisis de sus elementos y limitaciones. Actualidad Contable Faces, 9(12), 75-84.

Fraser, I. (1993). Triple-entry bookkeeping: A critique. Accounting and Business Research, 23(90), 151-158.

Galle, R. y Mucelli, U. (2015). Pasado, presente y futuro de la contabilidad de gestión. Revista Institucional de la Facultad de Ciencias Económicas, 9(5). Recuperado de: http://sedici.unlp.edu.ar/handle/10915/43826

Glover, J. (2018). Yuji Ijiri: sobre contabilidad. Accounting, Economics, and Law: A Convivium. 8(1), 1-4. doi: https://doi.org/10.1515/ael-2018-0004

Ijiri, Y. (1986). A framework for triple-entry bookkeeping. The Accounting Review, LXI (4), 745-759. 
Kaplan, R. (1984). The evolution of management accounting. The Accounting Review, 59(3), 390-418.

Lizcano, J. (1982). La dimensión integral de la empresa. Un modelo para su evaluación y representación contable (Tesis doctoral), Universidad Autónoma de Madrid, Madrid. Recuperada de: https://repositorio.uam.es/ handle/10486/2359

Lozano, C. (2019). Partida multidimensional para toma de decisiones de Contadores Públicos de Huancayo, Huánuco y Huancavelica, 2014 (Tesis doctoral). Universidad Peruana los Andes, Huancayo. Recuperada de: http://repositorio.upla.edu.pe/bitstream/handle/ UPLA/836/T037_20097365_D.pdf?sequence=1\&isAllowed $=\mathrm{y}$

Lozano, C. y Lozano, P. (2012). ¿Eleusis o Mayeusis del árbol contable? Arjé de partida triple y partida multidimensional de $360^{\circ}$. VIII Congreso Iberoamericano de Administración Empresarial y Contabilidad y VI congreso Iberoamericano de Contabilidad de Gestión. Organizado por la Facultad de Administración y Contabilidad de la Pontifica Universidad Católica del Perú.

Mejía, E. et al. (2005). Contabilidad y racionalismo crítico. Cali: Editorial Universidad Libre Seccional Cali.

Mejía, E., Montes, C., y Dávila, G. (2011). Introducción a la propuesta contable de García-Casella. Cuadernos de Contabilidad, 12(30), 127-164. Recuperado de: http:// www.scielo.org.co/scielo.php?script=sci_arttext\&pi$\mathrm{d}=$ S0123-14722011000100006\&lng=en\&tlng=es

Mejía, E. et al. (2013). Estructura conceptual de la teoría tridimensional de la contabilidad. Revista Contexto, 2, 49-70.

Melse, E. (2008), Accounting in three dimensions: a case for momentum revisited, Journal of Risk Finance, 9(4), 334350. doi: https://doi.org/10.1108/15265940810895007

Moreno, R. et al. (2017). La contabilidad triangular y su aporte a la toma de decisiones financieras- administrativas. Revista Académica-Investigativa de la Facultad Jurídica, Social y Administrativa, 3(5), 20-24. Recuperado de https://revistas.unl.edu.ec/index.php/suracademia/article/view/256

Ortiz, J. (2013). ¿Es necesaria y posible una teoría general para estructurar la ciencia contable? Revista Facultad de Ciencias Económicas: Investigación y Reflexión, 21(2), 115-141. Recuperado de https://www.redalyc. org/pdf/909/90930501008.pdf
Pei-Gin, H. (2013). Yuji Ijiri's thoughts as a possible solution for the recent revolutions in accounting standards- A focus on accounting measurements. Journal of Research in International Business and Management, 3(2), 38-52. Recuperado de: http://www.interesjournals.org/JRIBM

Porporato, M. (2015). Contabilidad de gestión para controlar o coordinar en entornos turbulentos: su impacto en el desempeño organizacional. Contaduría y Administración, 60(3), 511-534. doi: http://dx.doi.org/10.1016/j.cya.2015.02.002

Postner, H. (1988). Linkages between macro and micro business accounts: implications for economic measurement. The Review of Income and Wealth, 34(3), 1. doi: https://doi.org/10.1111/j.1475-4991.1988.tb00573.x

Sheposh, R. (2019). Dimensión. Salem Press Encyclopedia of Science, 1.

Staszkiewicz, P. W. (2011). Marco de entrada múltiple para informes financieros y de riesgos. Munich Personal RePEc Archive, (No 34903), 1-15. Recuperado de: https:// mpra.ub.uni-muenchen.de/34903/

Velásquez, N. (2019). Contabilidad Gerencial [versión PDF]. Recuperado de: http://repositorio.uladech.edu. pe/bitstream/handle/123456789/14784/CONTABILIDAD\%20GERENCIAL.pdf? sequence=3\&isAllowe$\mathrm{d}=\mathrm{y}$

Villaluenga, S. (2013). La partida doble y el cargo y data como instrumentos de un sistema de información contable y responsabilidad jurídica integral, según se manifiesta en fuentes documentales de la Catedral de Toledo (1533-1613). Revista de Contabilidad - Spanish Accounting Review,16(2), 126-135. doi: http://dx.doi. org/10.1016/j.rcsar.2013.07.002

Yavicoli, A. (2019). Fractales, patrones y dimensión. (Tesis doctoral). Universidad de Buenos Aires, Buenos Aires. Recuperada de: https://bibliotecadigital.exactas.uba. ar/download/tesis/tesis_n6626_Yavicoli.pdf 